\title{
Immediate Outcome and Predictors of Percutaneous Mitral Balloon Commissurotomy: A 5 Years Experience in Cardiac Center Ethiopia
}

\author{
Mohammed Bedru Sebah ${ }^{1}$, Kefelegn Dejene Tadesse ${ }^{1}$, Ali Dawed Mohammed ${ }^{1}$, \\ Fekede Agwar Debel ${ }^{1}$, Shibikom Tamirat Agonafir ${ }^{1}$, Azene Dessie Mengistu ${ }^{1}$, Jemal Haidar Ali ${ }^{2,}$, \\ ${ }^{1}$ Cardiac Center of Ethiopia, Addis Ababa, Ethiopia \\ ${ }^{2}$ Department of Public Health Nutrition, School of Public Health, Addis Ababa University, Addis Ababa, Ethiopia
}

Email address:

bedrusebah@gmail.com (M. B. Sebah),mohammedbedrus@yahoo.com (M. B. Sebah), kefruit@gmail.com (K. D. Tadesse), imranali0306@yahoo.com (A. D. Mohammed), kefedb@gmail.com (F. A. Debel), shisirr@gmail.com (S. T. Agonafir), admengistu2000@yahoo.com (A. D. Mengistu), hjemal@gmail.com (J. H. Ali)

${ }^{*}$ Corresponding author

To cite this article:

Mohammed Bedru Sebah, Kefelegn Dejene Tadesse, Ali Dawed Mohammed, Fekede Agwar Debel, Shibikom Tamirat Agonafir, Azene Dessie Mengistu, Jemal Haidar Ali. Immediate Outcome and Predictors of Percutaneous Mitral Balloon Commissurotomy: A 5 Years Experience in Cardiac Center Ethiopia. Cardiology and Cardiovascular Research. Vol. 3, No. 3, 2019, pp. 55-61. doi: $10.11648 /$ j.ccr.20190303.13

Received: July 23, 2019; Accepted: August 18, 2019; Published: September 4, 2019

\begin{abstract}
Percutaneous mitral balloon commissurotomy (PMBC) is the treatment of choice for rheumatic mitral stenosis with favorable anatomy and prevents complications inherent to a surgical procedure, while maintaining effectiveness. In view of this, we examined the immediate outcome and its predictors of patients undergoing PMBC in our Cardiac Center and availed evidence based information for future use of the treatment in Ethiopia. A retrospective data analysis of 330 patients with symptomatic severe mitral stenosis treated with PMBC using Inoue balloon in the cardiac center Ethiopia from 2014 to 2018 were assessed for their clinical outcome for those who presented for the first time in our cardiac center, Addis Ababa. Demographic, clinical, echocardiographic and hemodynamic data of all patients with symptomatic severe mitral stenosis were collected and analyzed. Of the 330 PMBC performed during the 5 years in the center, the majority had successful procedure $(97.88 \%)$ and optimal result $(92.42 \%)$. Twenty four $(7.2 \%)$ had complications of which $7(2.1 \%)$ had failed procedure and 9 (2.7\%) marked mitral regurgitation. Atrial fibrillation was observed in 67 (20.3\%). The mean mitral valve score (MVS) was $7.3 \pm 1.35$ ranged from 4 to 13 . A significant association of immediate outcome was observed with atrial fibrillation ( $\mathrm{AOR}=4.41 ; 95 \% \mathrm{CI}=1.51$ to 12.89$)$ and high mitral valve total Wilkins score ( $\mathrm{AOR}=0.25 ; 95 \% \mathrm{CI} 0.09$ to 0.70 ). The major predictors identified for poor outcome are atrial fibrillation and MVS. Percutaneous mitral balloon commissurotomy is a safe procedure with excellent immediate results signifying that it is a treatment of choice for heterogeneous group of patients with rheumatic mitral stenosis.
\end{abstract}

Keywords: Percutaneous Mitral Balloon Commissurotomy, Predictors, Cardiac Center Ethiopia

\section{Introduction}

Rheumatic fever (RF) has been the major culprit of rheumatic mitral stenosis (MS) and constitutes about 25 percent of all patients with rheumatic heart diseases [1-3]. In the developing countries, MS evolves more rapidly, possibly due to severe or repeated streptococcal infections, genetic influences, or economic conditions and results in characteristic changes of the mitral valve with the diagnostic features of thickening at the leaflet edges, fusion of the commissures, and chordal shortening and fusion. Subsequent scarring leads to the characteristic valve deformity with obliteration of the normal leaflet architecture by fibrosis, neovascularization and increased collagen and tissue cellularity $[4,5]$. 
Using Inoue balloon has long been considered an effective treatment modality among patients with favorable valve morphology, [6-9] and provides sustained hemodynamic improvement with minimal complication [10]. The procedure has been reported to be technically successful in over $90 \%$ of patients with its excellent long-term durability. In cases of unfavorable morphology (Wilkins score > 8) [11, 12] it postpones the need for surgical mitral valve replacement. Some of the unfavorable conditions include left atrial appendage clot and a redo-PMBC which may be associated with increased complications or incomplete success. In addition to these, difficult anatomy with intertribal sepal bulge, giant left atrium, calcific valve and involvement of submitral apparatus make left ventricular entry difficult during PMBC.

The most important steps in performing PMBC are atrial septal puncture and crossing the mitral valve [13-14] which are a fundamental knowledge to a technical success. The procedure is cost effective, less invasive and free from complications related to surgery and very rarely leads to pericardial tamponade [15-16]. Such intervention however, has not been done in the country and our cardiac center took the initiatives and performed PMBCs for patients referred from the entire country with heterogeneous presentations of severe MS. In view of these, we assessed the immediate outcomes and predictors of PMBC in patients with severe mitral stenosis and availed evidences based information for future use of the treatment in the country.

\section{Methods}

\subsection{Study Setting, Design, and Participants}

Children's heart fund of Ethiopia (cardiac center) placed in the center of the capital city of the country. It is a tertiary referral cardiac center for patients requiring cardiac intervention. The facility has 30 beds and gives 24 hour services for both children and adults as deemed necessary. It has a total of 13 heart team members that includes six cardiologists, two cardiac surgeons, two cardiac anesthesiologist, one cardiac intensivist and two percussionists. We used a single facility based observational study design from a dedicated database where, we retrospectively analyzed all 330 patients of both genders comprising all age groups from April 2014 to December 2018 who had PMBC for symptomatic severe mitral stenosis at the center.

\subsubsection{Inclusion Criteria}

All patients with symptomatic severe mitral stenosis including those with severe tricuspid regurgitation, moderate mitral regurgitation (MR), moderate aortic lesions, right and left ventricular systolic dysfunction, pregnancy, Lutembacher's syndrome, Mitral Stenosis with Left atrium (LA) Clot (type Ia), calcified valve with high mitral valve score and High-risk comorbid conditions like Thrombocytopenia, Hyperthyroidism were included in the study.

\subsubsection{Exclusion Criteria}

Major criteria for exclusion were the presence of left atrial cavity thrombus on transesophageal echocardiography (TEE), moderate to severe mitral regurgitation, bicommissural calcification, mitral valve area (MVA) $>1.5$ sqcm and previous mitral valve surgery.

\subsection{Ethical Issues}

The study was approved by the hospital's Institutional Human Research Review Committee. Prior to the PMBC intervention, informed written consent was also obtained from the respective guardians/parents of all patients. In addition, an assent was obtained in those patients who are above the age of 7 years.

\subsection{Pre-Procedural Evaluation}

Patients were subjected to an initial evaluation that included chest X-ray and surface electrocardiogram followed by a complete echo Doppler study within 24-48 hours prior to PMBC. Mitral valve anatomy was assessed according to Wilkin's criteria. Mean trans-mitral diastolic gradient was assessed by continuous wave Doppler. Mitral regurgitation was semi quantitatively graded from 0 to 4 by color flow mapping. The degree of commissural pathology is evaluated (in a parasternal short-axis view). After the procedure (within first 24hour) the commissures, mitral valve area and doppler data were examined.

\subsubsection{Clinical Presentation}

All patients had dyspnea ranged from New York Heart Association Functional classification (NYHA) functional class (II-IV). There were also patients with history of pregnancy, atrial fibrillation, previous embolization and previous commissurotomy.

\subsubsection{Procedure}

We employed the Inoue balloon catheter which has been used internationally as the first choice for significant MS for over 30 years because of its high technical feasibility and excellent results. The procedure was performed in the cardiac catheterization laboratory with the patient under either local or general anesthesia. One patient however had the procedure exclusively transthoracic echo guided because of accidental failure of fluoroscopy machine. Four patients had evidence of localized left atrial appendage thrombus on transesophageal echocardiographic examination detected during the procedure. All the procedures were performed aseptically through right femoral vein approach with surgical backup. Antibiotic prophylaxis was given for all patients. Septal puncture was performed using Brockenbrough technique [17].

Trans-septal puncture was the first and most crucial step of the procedure while monitoring arterial pressure by placing a pigtail catheter at the aortic sinus through the right femoral artery, usually through a 5 French sheath. After accessing the femoral vein, 8.5 French Mullins sheath was passed over an exchange length wire of 0.032-inch diameter into the 
superior caval vein. The Teflon guide wire was removed and Brockenbrough septal puncture needle was advanced in the Mullin's dilator. We preferred to use right anterior oblique (RAO) and left anterior oblique (LAO) views assisted by transthoracic echo for septal puncture. As soon as the whole assembly enters the left atrium, atrial entry was confirmed by recording the wave form of the left atrial pressure, by oximetry, or by injection of contrast into the left atrium. After confirming entry to the left atrium, 70-100 units of heparin per kilogram of body weight were given to minimize the risk of thrombosis, and the gradient across the mitral valve was measured by recording the left atrial pressure. The selfpositioning single balloon (Inoue balloon) was used for the commissurotomy in all patients. The upper limit of the balloon dilating diameter was chosen according to patients' height and echocardiographic parameter. Inflation was started at $2-4 \mathrm{~mm}$ less than the maximum diameter. When the hemodynamic results were suboptimal, the procedure was repeated by increasing the balloon diameter to the predetermined level. When the optimal hemodynamic results were not obtained at the balloon's maximum diameter, additional inflation was deferred. Once the balloon catheter's tip traversed the interatrial septum, the stiffening cannula for the central lumen was used to facilitate the passage of the balloon portion into the left atrium and subsequently maneuvered into the left ventricle (LV). The balloon was then inflated with diluted contrast material until the waist of the balloon disappeared. In routine practice, balloon size was chosen based on the patient's height using a simple Formula. The height-based formula estimated the sizes as being greater $(25.97 \pm .06 \mathrm{~mm})$ than the final balloon sizes used during PMBC $(24.10 \pm .09 \mathrm{~mm})$. The different techniques used to cross mitral valve orifice are depicted in Figure 1.
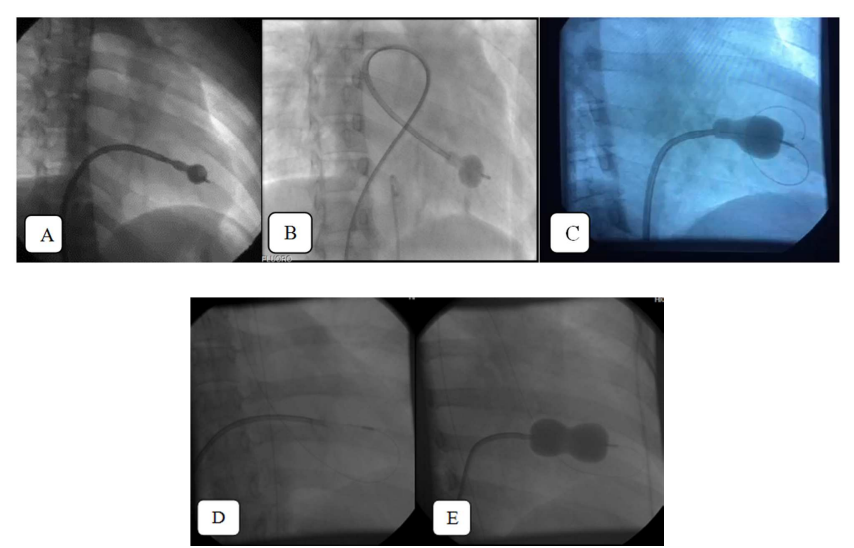

Figure 1. Techniques used to cross the mitral valve: (A) Standard techniques used to cross the mitral valve (B) Reverse loop techniques used to cross the mitral valve (C) Over-the-wire techniques used to cross the mitral valve (D) Veno-arterial loop techniques used to cross the mitral valve (E) Inflated balloon with central waist using veno-arterial loop.

Criteria for concluding the procedure included MVA $>1.5 \mathrm{~cm}^{2}$ or doubling of valve area, drop in mitral mean gradient by half, complete commissural opening in at least 1 commissure, and absence of an increase MR $>$ grade 2/4 and increment $>1$ from baseline Mitral regurgitation.

\subsubsection{Post-Procedural Evaluation}

After the procedure was completed all hemodynamic measurements were done in Cath lab and repeated again within the first 24 hours. The severity of the resultant mitral regurgitation, the peak and mean trans mitral gradient including pulmonary artery pressures were also measured.

\subsection{Data Collection and Method of Statistical Analysis}

Data on demographic, clinical, biological, echocardiographic and procedural information of patients were regularly recorded on standard forms and entered into a computer using excel version 10 and transported to STATA software version 13 for analysis.

Data were summarized using means and standard deviation (SD) for continuous variables, and frequency for categorical variables. Paired t-test was used to compare the mean differences of hemodynamic variables before and after valvuloplasty. Bivariate and multivariate logistic regression was used to identify factors that had significant association with the outcome. The tested variables were age group; gender; atrial fibrillation; stroke, left atrial size, total echocardiographic score and pulmonary hypertension. Those tested variables with a significance level of $20 \%$ in the bivariate analysis were further entered in the multiple logistic regression model to identify the predictors that retained their association with the outcome. A $\mathrm{p}$ value $\leq 0.05$ was considered statistically significant.

\section{Result}

As shown in Table 1, the mean age of patients was $28.79 \pm$ 10.58 years and ranged from 6 to 62 years. Most (72.12\%) of them were females and over a quarter $(27.88 \%)$ were males. Over three-fourth $(79.7 \%)$ had normal sinus rhythm and 67 $(20.3 \%)$ had atrial fibrillation, $37(11.2 \%)$ stroke, 73 $(22.12 \%)$ of them had spontaneous echo contrast. There were 13 pregnants $(3.9 \%)$ and four patients had previous mitral commissurotomy with mitral restenosis. Patient with severe TR and Moderate MR account for 72 (21.8\%) and 26 (7.8\%), respectively. Two Lutembacher's syndrome and four Mitral Stenosis with LA appendage clot confined to appendage were included in the study.

Table 1. Patient's clinical characteristics.

\begin{tabular}{ll}
\hline VARIABLES & N=330 \\
\hline Age, years & $28.79(6-62)$ \\
Female gender, n (\%) & $238(72.12)$ \\
Sinus rhythm, n (\%) & $263(79.70)$ \\
Stroke, n (\%) & $37(11.21)$ \\
Spontaneous echo contrast grade (I-IV), n (\%) & $73(22.12 \%)$ \\
Pregnant patients, n (\%) & $13(3.9)$ \\
Previous Commissurotomy, n (\%) & $4(1.2)$ \\
Severe Tricuspid Regurgitation, n (\%) & $72(21.8)$ \\
Moderate Mitral Regurgitation, n (\%) & $26(7.8)$ \\
Lutembacher'ssyndrome, n (\%) & $2(0.6)$ \\
Mitral Stenosis with LA Clot [type Ia]*, n (\%) & $4(1.2)$ \\
\hline
\end{tabular}

*LA (left atrium) appendage clot confined to appendage 
The procedural success rate $323(97.88 \%)$ while optimal result was $305(92.42 \%)$ with no hospital mortality rate. Prior to the procedure, the mean mitral valve area was $0.8 \pm$ $0.23 \mathrm{~cm}^{2}$, and after commissurotomy the mean mitral valve area improved significantly $\left(1.76 \pm 0.4 \mathrm{~cm}^{2 ;} \mathrm{p}<0.001\right)$. A reduction in transmitral mean gradient from $18.5 \pm 7.29$ $\mathrm{mmHg}$ to $6.15 \pm 2.75(\mathrm{p}<0.001)$ and the Wilkins Mitral valve score was $7.32 \pm 1.35$. At baseline, moderate MR was
$26(7.88 \%)$ and right after the procedure, MR doubled i.e 47 patients experienced moderate, 13 moderate to severe MR, and 2 patients experienced severe MR as shown in Table 2. On the other hand, the procedure was optimal in all pregnant women as well as in all patients with mitral valve restenosis and in $80 \%$ of calcified valves with Wilkins score $>8$ points.

Table 2. Pre-and post-procedural Echocardiographic characteristics.

\begin{tabular}{lll}
\hline Variables & Pre-procedural (n=330) & Post-procedural (n=330) \\
\hline Wilkins score, points & $7.32 \pm 1.35$ & NA \\
Wilkins score > 8 points, n (\%) & $40(12.12)$ & NA \\
Mitral valve area assessed by plannimetry, cm ${ }^{2}$ & $0.80 \pm 0.23$ & $1.76 \pm 0.40$ \\
Transmitral mean gradient, mmHg & $18.52 \pm 7.28$ & $6.35 \pm 3.92$ \\
TR gradient, mmHg ${ }^{* *}$ & $65.86 \pm 27.7$ & $40.68 \pm 15.95$ \\
Mitral Regurgitation, n & & $30(9.09)$ \\
No & $71(21.52)$ & $183(55.45)$ \\
Grade I & $213(64.55)$ & $55(16.67)$ \\
Grade I-II & $20(6.06)$ & $47(14.24)$ \\
Grade II & $26(7.88)$ & $13(3.94)$ \\
Grade II-III & 0 & $2(0.61)$ \\
Grade III and IV & 0 & \\
\hline
\end{tabular}

$\mathrm{N}=$ number of patients; $\mathrm{NA}=$ not available

**data is available for 314 and 305 patients for pre/post-procedure, respectively

As shown in Table 3, MVS and atrial fibrillation were the major predictors associated with the immediate outcome of PMBC. On the other hand, PHTN, stroke, age, gender and left atrium size had no significant association with the outcome. The immediate outcome was inversely proportional to the MVS ( $\mathrm{AOR}=0.25 ; 95 \% \mathrm{CI}=0.09-0.70)$ while the odds of atrial fibrillation was 4.4 times more likely to have an excellent outcome.

Table 3. Factors associated with immediate outcome of the PMBC at the center.

\begin{tabular}{|c|c|c|c|c|c|}
\hline \multicolumn{6}{|l|}{ Multivariate Analysis } \\
\hline Outcome & OR & SE & $\mathbf{Z}$ & $\mathbf{P}>\mathbf{Z}$ & [95\% Conf. Interval] \\
\hline Pulmonary hypertension & 0.38 & 0.192 & -1.91 & 0.056 & $0.14-1.02$ \\
\hline Mitral Valve score & 0.25 & 0.131 & -2.63 & 0.008 & $0.09-0.70 *$ \\
\hline Atrial Fibrillation & 4.41 & 2.414 & 2.71 & 0.007 & $1.51-12.89 *$ \\
\hline Stroke & 1.17 & 0.729 & 0.26 & 0.798 & $0.35-3.96$ \\
\hline Age group & 0.82 & 0.465 & -0.36 & 0.722 & $0.27-2.49$ \\
\hline Left Atrium & 0.92 & 0.460 & -0.17 & 0.867 & $0.34-2.45$ \\
\hline
\end{tabular}

*significant; $\mathrm{OR}=$ odds ratio; $\mathrm{SE}=$ standard error

As shown in Table 4, twenty-four (7.2\%) of the patients had complications. Of these, one patient developed large pericardial effusion amounting to cardiac tamponade which was relieved with tapping and procedure completed before discharge. Mild pericardial effusion was seen in $5(1.5 \%)$ patients and PMBC was deferred in the three of these patients (one of them went for surgery and two repeat procedures were done successfully before discharge). New onset atrial fibrillation was encountered in $2(0.6 \%)$ patients during and immediately after PMBC. One patient $(0.3 \%)$ developed cerebrovascular accident with significant neurological deficits during procedure, which recovered gradually, and two patients had a transient ischemic attack. As expected, all had atrial fibrillation, but none had evidence of left atrial thrombus on TEE examination, which was done prior to the procedure.

Peripheral arterial embolization to the left femoral artery occurred in 1 patient $(0.3 \%)$ who later recovered with medical treatment. Electrocardiogram (EKG ) change and typical anginal chest pain was present in one patient during procedure immediate coronary angiogram didn't show obstructive coronary disease. Although one patient develops cardiac arrest during the balloon inflation, she recovered after prolonged chest compression. Significant MR increment by more than one degree and greater than moderate $\mathrm{MR}$ or Severe MR was observed in 9 (2.7\%) patients. While acute severe mitral regurgitation requiring mitral valve surgery was noticed in one $(0.3 \%)$ and the rest were managed medically and were followed up. Operative report was available in a patient who underwent emergent mitral valve repair due to severe mitral regurgitation. The mechanism of mitral regurgitation was identified to be secondary to torn valve leaflets. Most importantly, no death has occurred in this study. 
Table 4. Complications related to PMBC procedure.

\begin{tabular}{ll}
\hline In-hospital events & N (\%) \\
\hline Pericardial tamponade & $1(0.3)$ \\
Pericardial Perforation/mild effusion & $5(1.5)$ \\
New onset atrial fibrillation & $2(0.6)$ \\
Stroke & $3(0.9)$ \\
Peripheral arterial embolization & $1(0.3)$ \\
Transient ischemia with EKG change & $1(0.3)$ \\
Cardiac arrest & $1(0.3)$ \\
Grade 3 mitral regurgitation & $9(2.7)$ \\
Emergent mitral valve repair & $1(0.3)$ \\
Mortality & 0 \\
\hline
\end{tabular}

In our series, there were a total of 7 (2.12) unsuccessful procedures due to failure to cross the mitral valve, of theses, 5 of them underwent elective mitral valve replacement and there were peculiar echocardiographic features for inability to cross the mitral valve as depicted in Figure 2.

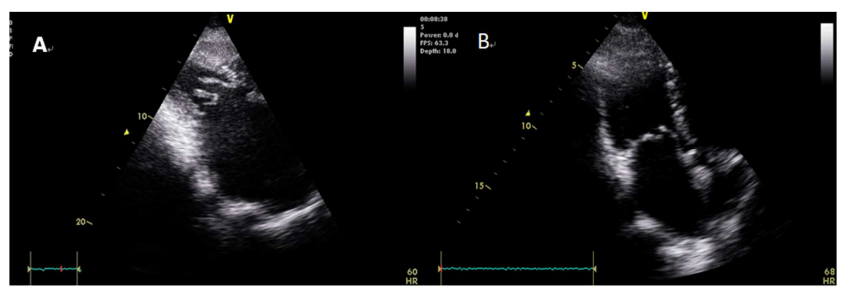

Figure 2. Reasons for inability to cross the mitral valve orifice: (A) Tunnel mitral valve due to severe subvalvular disease (B) Fixed posterior mitral valve leaflet with eccentric orifice abating the body of anterior leaflet.

\section{Discussion}

The present study has addressed an important issue of practical importance in the management of symptomatic severe mitral stenosis in Ethiopian context and yielded an excellent result which is analogues with a number of previous studies done elsewhere that documented satisfactory immediate and long-term outcome [18-22]. The dominant mechanism of commissurotomy is by splitting the commissures and giving non fused, wide open mitral valve [23].

The incidence of significant MR requiring urgent MVR was lowest in our study with fair results compared to other report [24]. The reason for a lower incidence of significant MR after procedure may be due to the fact that the balloon dilatation was started from low pressure inflation and gradually increased to optimal size to achieve adequate result.

It is evident that, the outcomes of PMBC in patients of RMS in atrial fibrillation and high mitral valve score are considered to be inferior to outcomes of patient in rheumatic mitral stenosis in normal sinus rhythm and total echocardiographic scores $<8$. Our study also documented this close association in parallel with current evidence. Likewise, some studies reported no correlation between successful PMBC and patient age, gender, pulmonary hypertension and stroke $[25,26]$. A score $>8$ does not preclude PMBC, but is associated with less optimal results. Commissural calcification is a strong predictor of adverse outcomes of
PMBC as well as of the occurrence of severe MR as a major complication of PMBC [26]. The development of atrial fibrillation (AF) is common sequelae in patients with long standing MS, and it is associated with hemodynamic and clinical decompensation. Leon et al have pointed out that the presence of $\mathrm{AF}$ is associated with inferior immediate and long-term outcome following $\mathrm{PMBC}$ in patients with $\mathrm{AF}$ [27]. Interstingly, the incidence of AF in our study is $20.3 \%$ and was associated with poor immediate result.

The difficulties we encountered during PMBC were multifold and ranged from intricate anatomy like intunnel mitral valve orifice, eccentric mitral valve orifice, thick interatrial septum, and bulging interatrial septum and severe under estimated sub valvular disease which made left ventricular entry during PMBC difficult.

On the other hand, of the 7 encountered failed patients, 6 of them were in the first 150 cases suggesting that the technical refinements on the immediate outcome of PMBC was not full-fledged though later the technical refinements significantly improved and in the same breath, complication markedly reduced when compared with our initial operating experience.

We advocate revision of septal puncture if the first attempt at left ventricular entry fails and proceed with a second attempt which is a standard modified technique like reverse loop to be used. If the aforementioned attempts fail again, then veno-arterial looping can be considered. For most PMBC cases, the preferred puncture site for trans septal access to the left atrium is in the posterior, more inferior region of the fossa ovalis. Many operators rely primarily on fluoroscopic guidance, though Transthoracic echocardiography (TTE) guidance can also be an alternative since it is helpful, especially in patients with a large atrium or unusual morphology of the interatrial septum (IAS).

The incidence of cardiac perforation has been reported to vary between 1.5 to $4.7 \%$ percent during $\mathrm{PMBC}$ as the main complications of trans-septal puncture are cardiac tamponade and hemopericardium by some authors [28, 29]. Similarly comparable result was also found in our series.

The presence of a thrombus in either the left atrium or left atrial appendage is considered as a relative contraindication to PMBC. If a thrombus is detected, the procedure should be postponed until the thrombus resolves on future TEE evaluation. If the thrombus does not resolve, surgery is considered as the preferred treatment option. However, some interventionists have shown that it is feasible to perform PMBC using a modified Inoue technique in selected patients with laminar thrombus confined to the left atrial appendage, particularly after 4 to 6 weeks of effective anticoagulation treatment [30]. In our study, four patients with organized left atrial appendage thrombus were seen and we were able to do a successful procedure with the Inoue balloon after adequate anticoagulation for more than 6 weeks using standard technique which makes this study peculiar. One of them had recurrent hospital admission for congestive heart failure and was on anticoagulation for more than 18 month and unable to get open heart surgery because of very limited service. At present, we do not recommend this procedure as a 
routine for all patients with organized left atrial thrombus. Nonetheless, when the condition of the patient is a life threatening, it is a possible alternative to open mitral commissurotomy to save life.

The present study is the first of its kind to report the outcome of Percutaneous mitral balloon commissurotomy in the Ethiopian settings referred from every part of the country which involved all age group of patient with different clinical presentations including moderate MR, Severe TR, pregnancy, highly calcified valve and Lutembacher's syndrome. Other than these, the study also identified the important predictors of immediate outcome.

\section{Conclusion}

Percutaneous mitral balloon commissurotomy is an important treatment option. It is a safe procedure with excellent immediate results signifying that it is a treatment of choice for heterogeneous group of patients with rheumatic mitral stenosis. Exceptional immediate outcome was observed among those who had lower echocardiographic scores and those who were sinus rhythm at presentation. In addition to these, patients with mitral restenosis, pregnancy and majority of calcified valves had optimal result irrespective of age. PMBC in patient with Lutembacher's syndrome, moderate mitral regurgitation, severe tricuspid regurgitation (TR), mitral stenosis with localized LA appendage clot and high-risk comorbid conditions like thrombocytopenia is feasible and showed satisfactory result in our study population.

\section{Recommendation}

In order to obtain a good functional outcome, it is essential to target the above identified predictors through appropriate patient selection based on imaging including a complete echo Doppler study preceding the procedure. Other than these, clinical and biochemical data, procedural technique, and quality of the initial result are of paramount importance to excellent immediate results.

\section{Authors Contribution}

BM, DK, DA, FA and ST organized the study when working for CCE, conducted statistical analysis and drafted the MS. While JHA contributed to data interpretation and revising the MS for intellect content. All authors read and approved the submitted manuscript.

\section{Conflicts of Interest}

The authors have no competing interests, funding, or financial relationships to disclose.

\section{Acknowledgements}

The authors would like to thank the entire team of Cardiac
Center Ethiopia (CCE) for their all rounds support and Chain of hope England for donating most of the Inoue balloon used for performing the procedure.

\section{Funding}

All the necessary expenses were covered by the authors.

\section{Availability of Data and Material}

The institutional review board of the hospital does not allow to share original data unless indicated in the approval process.

\section{References}

[1] Lung B, Baron G, ButchartEG, et al. A prospective survey of patients with valvular heart disease in Europe: the Euro Heart Survey on Valvular Heart Disease. Eur Heart J 2003; 24: 1231-43.

[2] Carapetis JR, SteerAC, Mulholland EK, Weber M. The global burden of group A streptococcal diseases: a population based study. Lancet Infect Dis 2005; 5: 685-94.

[3] Marijon E, Ou P, Celermajer DS, et al. Prevalence of rheumatic heart disease detected by echocardiographic screening. N Engl J Med 2007; 357: 470-6.

[4] Rowe JC, Bland EF, Sprague HB, White PD. The course of mitral stenosis without surgery: ten- and twenty-year perspectives. Ann Intern Med 1960; 52: 741-9.

[5] Strasser T, Dondog N, El Kholy A, et al. The community control of rheumatic fever and rheumatic heart disease: report of a WHO international cooperative project. Bull World Health Organ 1981; 59: 285.

[6] Borger MA, CarrelTP, DeBonis M, et al. The Joint Task Force on the Management of Valvular Heart Disease of the European Society of Cardiology (ESC) and the European Association for Cardio-Thoracic Surgery (EACTS). Guidelines on the management of valvular heart disease (version 2012). Eur Heart J 2012; 33: 2451-96.

[7] Bonow RO, Carabello BA, Chatterjee K, et al., American College of Cardiology/American Heart Association Task Force on Practice Guidelines. 2008 Focused update incorporated into the ACC/ACC 2006 guidelines for the management of patients with valvular heart disease. J Am Coll Cardiol 2008; 52: e1-142.

[8] Vahanian A, Baumgartner H, Bax J, et al. Guidelines on the management of Valvular Heart Disease of the European Society of Cardiology. Eur Heart J 2007; 28: 230-68.

[9] Inoue K, Owaki T, Nakamura T, et al. Clinical application of transvenous mitral commissurotomy by a new balloon catheter. J Thorac Cardiovasc Surg 1984; 87: 394-402.

[10] Nobuyoshi M, Arita T, Shirai SI, et al. Percutaneous balloon mitral valvuloplasty a review. Circulation. 2009; 119 (8): e211ee219.

[11] Nair KK, Pillai HS, Thajudeen A, et al. Comparative study on safety, efficacy, and midterm results of balloon mitral valvotomy performed with triple lumen and double lumen mitral valvotomy catheters. Cathet Cardiovasc Interv. 2012; 80 (6): 978e986. 
[12] Ben-Farhat M, Betbout F, Gamra H, et al. Predictors of longterm event-free survival and of freedom from restenosis after percutaneous balloon mitral commissurotomy. Am Heart J. 2001; 142 (6): 1072e1079.

[13] Vahanian A. Percutaneous mitral commissurotomy: an effective treatment in 'ideal' candidates whatever the approach. Eur Heart J 1997; 18 (11): 1689-90.

[14] Vahanian A, Iung B. Percutaneous mitral balloon commissurotomy: a useful and necessary treatment for the western population. Eur Heart J 2000; 21 (20): 1651-2.

[15] Thomas T, Ananthakrishna R, Chikkabasavaiah NA, Basavappa R. Application of a novel percutaneous transluminal mitral commissurotomy technique in deformed mitral valve. BMJ Case Rep 2011; 2011.

[16] Ootaki Y, Kozawa S, Asada T, Mukohara N, Higami T, Iwahashi K. Rupture of the papillary muscle after percutaneous transvenous mitral commissurotomy (PTMC) a case report. Nihon Kyobu Geka Gakkai Zasshi 1997; 45 (10): $1738-42$.

[17] Earley MJ. How to perform a transseptal puncture. Heart. 2009; 95 (1): 85e92.

[18] Nishimura RA, Holmes DRJr, Reeder GS. Percutaneous balloon valvotomy. Mayo Clin Proc 1990; 65: 198-220.

[19] Complications and mortality of percutaneous balloon mitral commissurotomy: a report from the National Heart, Lung, and Blood Institute Balloon Valvotomy Registry. Circulation 1992; 85: 2014-24.

[20] Bouleti C, Iung B, Himbert D, et al. Reinterventions after percutaneous mitral commissurotomy during long-term follow-up, up to 20 years: the role of repeat percutaneous mitral commissurotomy. Eur Heart J 2013; 34: 1923.

[21] Kim D, Chung H, Nam JH, et al. Predictors of Long-Term Outcomes of Percutaneous Mitral Valvuloplasty in Patients with Rheumatic Mitral Stenosis. Yonsei Med J 2018; 59: 273.
[22] Song H, Kang DH, Kim JH, et al. Percutaneous mitral valvuloplasty versus surgical treatment in mitral stenosis with severe tricuspid regurgitation. Circulation 2007; 116: I246.

[23] Block PC, Palacios IF, Jacobs MC, Fallon JT. Mechanisms of percutaneous mitral valvotomy. Am J Cardiol 1987; 59: 31823.

[24] Kim MJ, Song JK, Song JM, et al. Long-term outcomes of significant mitral regurgitation after percutaneous mitral valvuloplasty. Circulation 2006; 114: 2815.

[25] Sutaria N, Northridge DB, Shaw TRD. Significance of commissural calcification on outcome of mitral balloon valvotomy. Heart 2000; 84: 398-402.

[26] Padial LR, Freitas N, Sagie A, et al. Echocardiography can predict which patients will develop severe mitral regurgitation after percutaneous mitral valvotomy. J Am Coll Cardiol 1996; 27: $1225-31$.

[27] Wilkins GT, Weyman AE, AbascalVM, Block PC, Palacios IF. Percutaneous balloon dilatation of the mitral valve: an analysis of echocardiographic variables related to outcome and the mechanism of dilatation. Br Heart J. 1988; 60 (4): $299 \mathrm{e} 308$.

[28] Friedrich SP, Berman AD, Baim DS, Diver DJ. Myocardial perforation in the cardiac catheterization laboratory: incidence, presentation, diagnosis, and management. Cathetcardiovasc Diagn 1994; 32: 99-107.

[29] Teresa S M Tsang, William K Freeman, Marion E Barnes Ms, Guy S Reeder, Douglas L Packer, James B Sward. Rescue Echocardiographically Guided Pericardiocentesis for cardiac perforation complicating catheter-based procedures- the Mayo Clinical Experience JACC 1998: 32 (5): 1345-50.

[30] Hung J-S. Mitral stenosis with left atrial thrombi: Inoue balloon catheter technique. In: Cheng TO, editor. Percutaneous Balloon Valvuloplasty. New York, NY: IgakuShoin, 1992: 280-93. 